\title{
FIBROCYSTIC DISEASE OF THE PANCREAS IN THE NEWBORN
}

\author{
BY \\ ALBERT E. CLAIREAUX \\ From the Bernhard Baron Memorial Research Laboratories, Queen Charlotte's Maternity Hospital, \\ and the Institute of Obstetrics and Gynaecology, University of London
}

(RECEIVED FOR PUBLICATION SEPTEMBER 1, 1955)

Fibrocystic disease of the pancreas in the newborn infant is usually recognized by the development of intestinal obstruction as a result of meconium ileus (Andersen, 1938). According to Bodian (1952), a certain number of infants affected by fibrocystic disease develop severe intestinal lesions during intrauterine life. The abnormal consistence of the mucus produced in the alimentary tract causes inspissation of meconium and leads to intestinal obstruction, perforation or even atresia of the bowel. In the older infant the disease is more likely to take the form of a severe nutritional disorder which is liable to be complicated by respiratory infection. In a number of patients the respiratory disorder may dominate the clinical picture (Farber, 1944). Intestinal obstruction is rare in this group of patients, but it has been reported by Levy (1951) and by Fisher (1954) as a late complication of fibrocystic disease.

On the other hand, a congenital disorder such as fibrocystic disease of the pancreas is liable to be undetected in the neonatal period unless it appears as meconium ileus. Bodian (1952) states that in his series no children died in the first days of life with fibrocystic disease other than those with meconium ileus. He was thus unable to compare the distribution of intestinal lesions in infants of this age group with and without meconium ileus. In this laboratory we have examined sections of the pancreas obtained from a consecutive series of 500 necropsies on newborn infants without finding a single example of fibrocystic disease.

Recently, however, a case was encountered of an infant who died from anoxia during delivery. At post-mortem examination severe fibrocystic disease of the pancreas was found and lesions were also visible in the liver. There was no evidence of intestinal obstruction and the large bowel was full of meconium. This appears to be the first recorded example of fibrocystic disease of the pancreas without an intestinal lesion in a newborn infant.

\section{Case Report}

The mother was a healthy woman aged 29 years and this was her second pregnancy. Her first pregnancy in 1953 resulted in the birth of a boy who is now alive and well. Delivery was effected by Caesarean section at 37 weeks on account of placenta praevia. She developed acute nephritis two months after delivery but recovered fully.

During the pregnancy she was quite well. She was Group $\mathrm{O} R \mathrm{R}$ positive and no antibodies were present in the serum. The haemoglobin was $12.7 \mathrm{~g}$. $\%$ and red cells were 4.3 million per c.mm. A chest radiograph was normal and the Wassermann and Kahn tests were negative. Her blood pressure was $120 / 90 \mathrm{~mm}$. $\mathrm{Hg}$ and no abnormal constituents were present in the urine. No abnormality was detected at routine ante-natal examination.

Labour began spontaneously on February 26, 1955, seven days after the estimated date of delivery. The patient's blood pressure had risen to $140 / 90 \mathrm{~mm}$. $\mathrm{Hg}$. The urine was still clear. The foetus presented by the vertex. A slight intrapartum haemorrhage occurred and signs of foetal distress became evident 12 hours after the onset of labour. The foetal heart rate fell to $80 / \mathrm{min}$. and meconium was present in the liquor. On account of the foetal distress forceps delivery was carried out under local anaesthesia and when the cervix was fully dilated episiotomy was performed. The head was delivered slowly but the baby was now very limp and covered with meconium. The apex beat was not perceptible at delivery and all attempts at resuscitation failed. The placenta weighed $624 \mathrm{~g}$. and was perfectly normal. The puerperium was uneventful.

Necropsy. The body was that of a well developed female infant weighing $2,975 \mathrm{~g}$. No congenital abnormality was found on external examination.

The falx and tentorium were intact. The brain was firm and no lesion was found on slicing.

The larynx, trachea and bronchi were healthy. No free fluid was found in the pleural sacs. The lungs were intensely congested and subpleural petechial haemorrhages were present over their surface. There was evidence of aspiration of liquor amnii. The heart and great vessels were normal.

The peritoneum was healthy and no lesions were 
present in stomach or bowel. The content of the small intestine was yellow and semi-fluid. There was no overdistension. The colon was loaded with meconium, which was rather yellow instead of the usual dark green. The consistence of the meconium was normal. The liver $(140 \mathrm{~g}$.) was large and congested. The capsule was smooth but numerous small white flecks were seen on the liver surface just beneath. Similar flecks were seen on the cut surface of the organ when sliced. The flecks were again near the capsule. The gall bladder was extremely small and narrow. It contained some yellow mucus. The bile ducts were patent.

The pançreas was firm and nodular. The surface was very irregular and on slicing greenish-white material could be expressed from the cut surface. This material looked like pus. No macrocysts were seen.

The spleen was small and firm. The lymph nodes were normal. The ovaries, tubes, uterus, thyroid, thymus, pituitary and suprarenal glands showed no abnormality.

Material expressed from the pancreas was examined bacteriologically. Smears showed numerous pus cells but no organisms. All cultures were sterile.

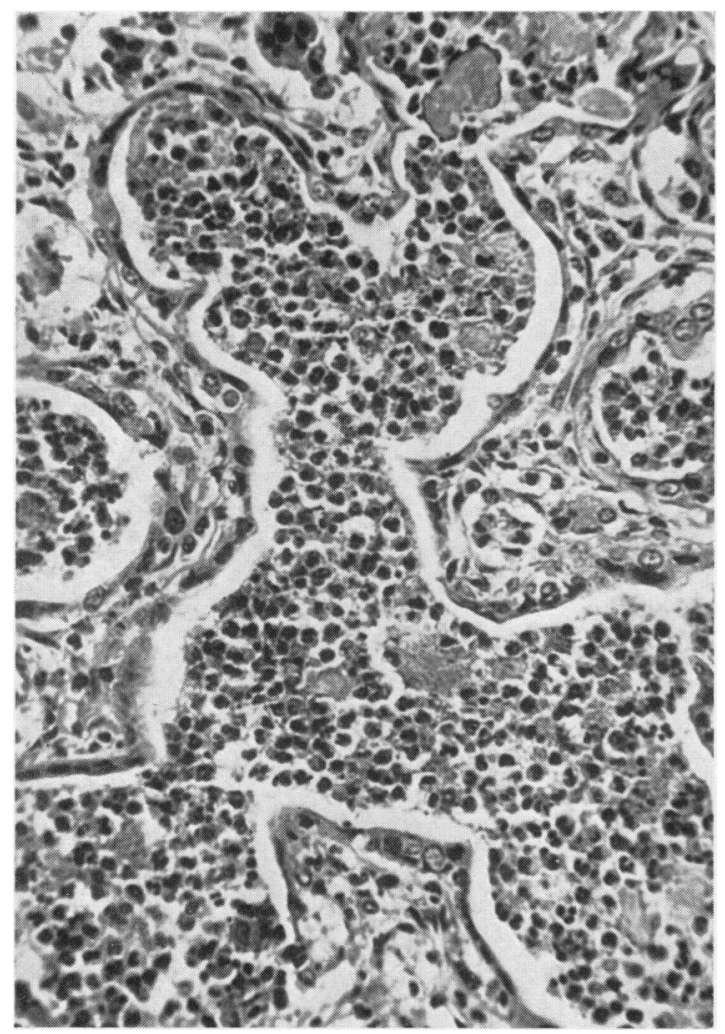

Fig. 1.-Pancreas $\times 250$. Grossly distended pancreatic duct filled with acidophil debris and polymorphonuclear leucocytes. The smaller ducts are surrounded by proliferated fibrous tissue. (All sections stained with haematoxylin and eosin.)
Histology. The pancreas showed a great increase in connective tissue with a marked atrophy of the acinar tissue. The degree of pathological change varied greatly from lobule to lobule. In some lobules the acini had almost completely disappeared and in these areas the gland was composed of enormously dilated ducts filled with amorphous, slightly eosinophil debris and polymorphonuclear leucocytes (Fig. 1). In some places the duct walls had undergone necrosis and pools of inspissated secretion had formed. The ducts were surrounded by dense fibrous tissue. It was presumably the material from these dilated ducts which was expressed when the pancreas was cut during post-mortem examination. Elsewhere the destructive process was less severe. The acinar tissue was still recognizable and in a very few areas appeared almost normal. The islets of Langerhans showed no change. Even in the less affected lobules, however, many acini were distended and filled with eosinophilic material which had the appearance of inspissated secretion (Fig. 2). The affected structures were surrounded by fibrous tissue which was infiltrated with lymphocytes and mononuclear cells. There was little evidence of necrosis in these areas, but again

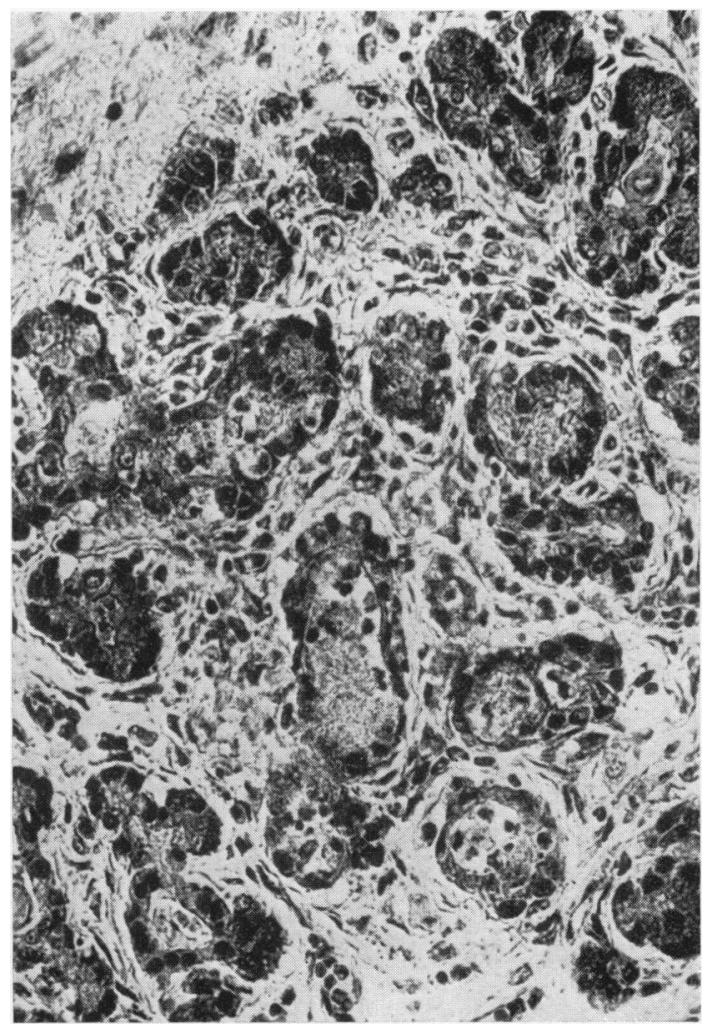

FIG. 2.-Pancreas $\times 250$. Dilated acini filled with inspissated secretion. There is pronounced periacinar fibrosis but less evidence of inflammatory cell infiltration in this area. 
infiltration with polymorphonuclear leucocytes was observed.

The liver showed focal biliary cirrhosis (Fig. 3). The portal tracts chiefly affected were those immediately beneath the capsule and corresponding to the white flecks observed on macroscopic examination. Elsewhere, especially toward the centre of the organ, the bile ducts and portal tracts had a normal appearance. In the cirrhotic areas there was proliferation of small bile ducts and an increase in the fibrous tissue stroma in the portal tracts. The bile ducts were distended and contained some amorphous material which stained brick-red with haematoxylin and eosin (Fig. 4). The material was probably inspissated mucus. The portal tracts were infiltrated with polymorphonuclear leucocytes and lymphocytes and mononuclear cells.

The lumen of the gall bladder was filled with thick mucus. The lining cells were of the tall columnar type. The wall was congested and fibrosed and no dilated mucous glands were seen.

The lungs were slightly expanded as a result of excessive aspiration of liquor amnii. Many alveoli and alveolar ducts contained cornified cells. The lungs were congested. The bronchi in the lung roots showed an abnormality of the mucous glands (Fig. 5). These glands

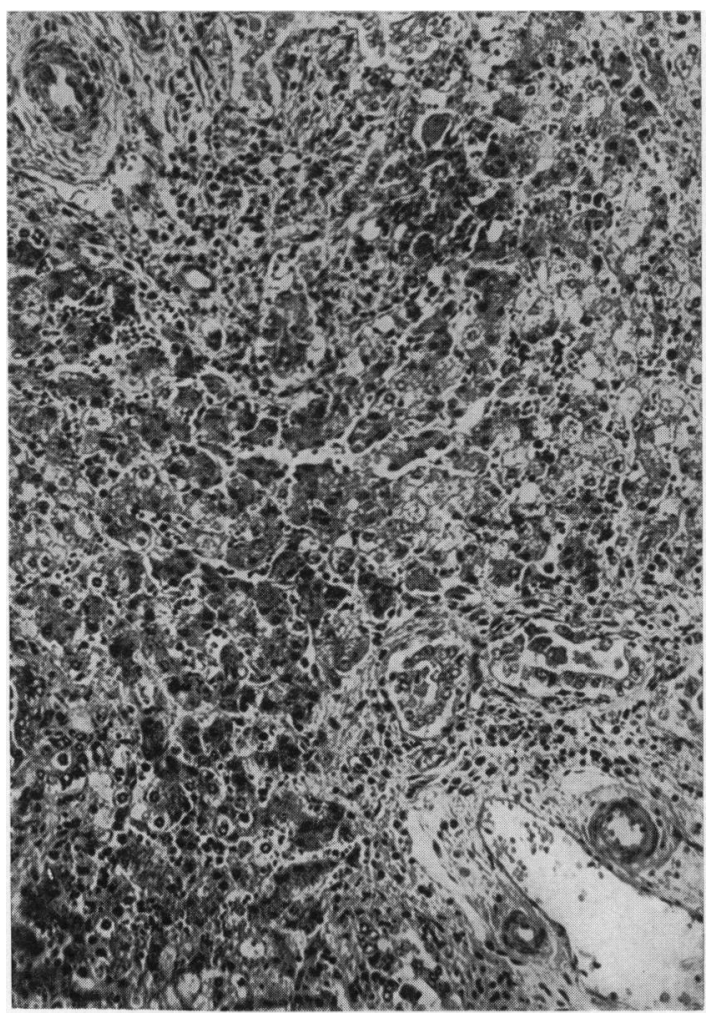

FIG. 3.-Liver $\times 100$. Focal biliary cirrhosis. The portal tracts show proliferation of small bile ducts, increase in fibrous tissue and infiltration with inflammatory cells. were distended with inspissated secretion. The bronchial epithelium had a normal appearance.

The parotid gland appeared normal. The submandibular salivary glands showed moderate dilatation of groups of acini which contained mucus. The ducts contained mucous secretion. The changes were not severe. No sections were taken from the sublingual glands.

Sections were examined from the stomach, duodenum, ileum and colon. The gastric mucosa showed fairly severe post-mortem change. A few glands were distended with thick mucus, but they were the exception. Some of Brunner's glands in the duodenum were dilated and contained inspissated mucus, but the lesion was not pronounced. The ileum showed post-mortem change of the mucosa. A few dilated glands were present. The mucous membrane of the colon was healthy. The villi appeared normal. Many goblet cells contained mucus, but there was no glandular dilatation (Fig. 6).

The spleen, kidneys and suprarenal glands showed no pathological change.

\section{Discussion}

This infant obviously had severe fibrocystic disease of the pancreas. The criteria laid down by

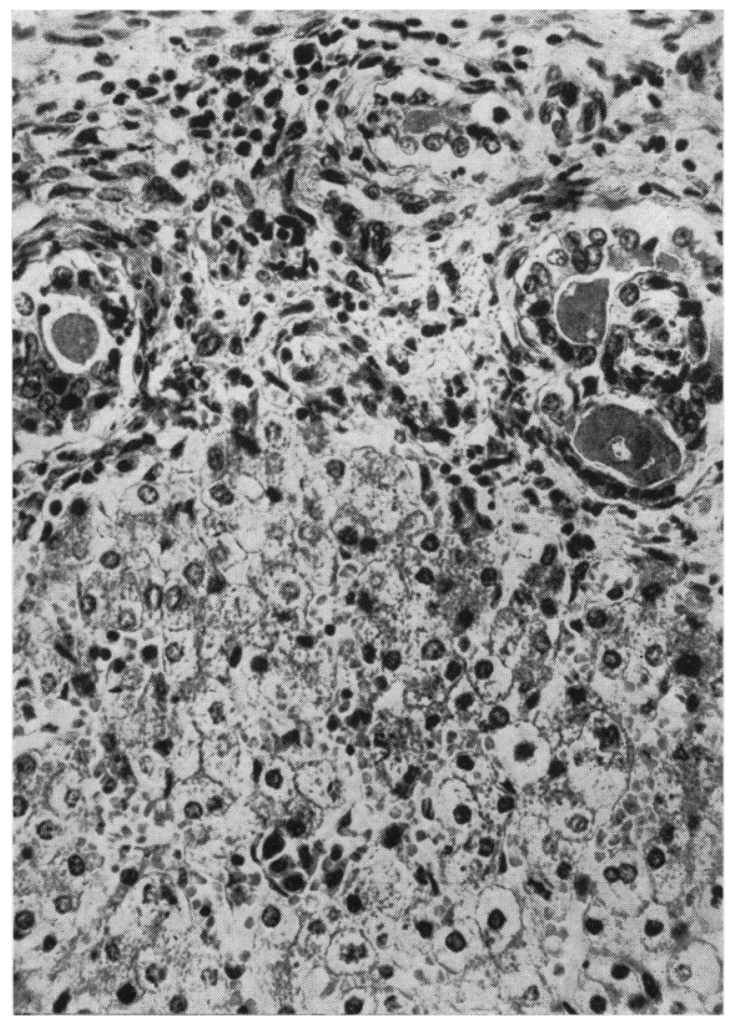

FIG. 4. - Liver $\times 250$. Portal tract showing distended bile ducts containing acidophil secretion. There is an increase in fibrous tissue which is infiltrated with polymorphonuclear leucocytes and lymphocytes. 


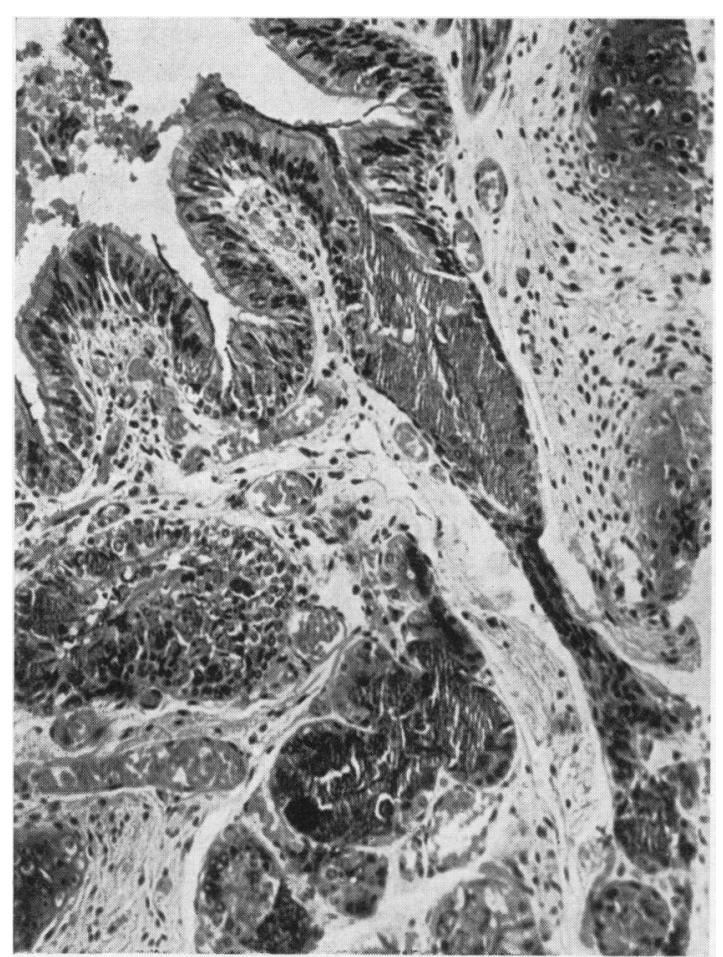

FIG. 5.-Lung $\times 100$. Portion of wall of major bronchus showing dilatation of mucous gland which is packed with secretion. The gland on the right is emptying viscid mucus into the bronchial lumen.

Bodian (1952) were all present. There was an excessive quantity of abnormal secretion, atrophy of exocrine tissue and a fibroblastic reaction and fibrosis of the stroma. Some pancreatic lobules were much more severely affected than others and this is characteristic of the disease. In some areas there was almost complete fibrosis with disappearance of exocrine parenchyma and in others only mild dilatation of the acini was found. However, the pancreas in this case presented some rather unusual features. The lesion was very far advanced at this stage of development and the presence of such large numbers of polymorphonuclear leucocytes was most extraordinary. Farber (1944) found large mononuclear cells and evidence of lymphocytic infiltration in some of his material, but 'neither necrosis nor acute inflammatory changes were encountered'. Inflammatory infiltration was not a feature of the series reported by Bodian (1952). Both necrosis and severe inflammatory reaction occurred in this pancreas. The ducts were enormously distended with inspissated secretion and many were packed with polymorphs. The duct walls were also necrosed.

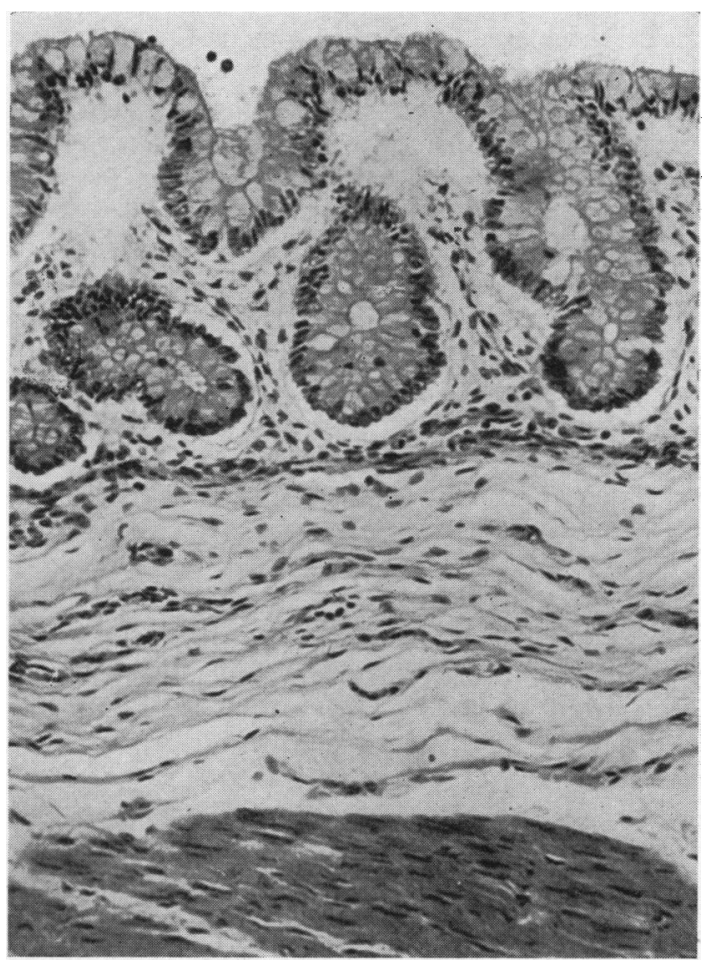

FIG. 6.-Colon $\times 150$. Mucous membrane is healthy. Many of the goblet cells in the epithelium contain mucus, but there is no glandular dilatation.

The cause of this inflammatory reaction is obscure. All attempts at recovering an organism failed. Bodian (1952) suggested that retained secretion may cause pressure atrophy of the exocrine tissue and during this process intracellular substances are liberated. These substances are believed to be irritant and produce a fibroblastic reaction and fibrosis. If such a process does occur it may explain the inflammatory changes in this case.

This question of inflammatory change is also connected with the development of the pancreatic lesion in its early stages. Farber (1944) found only inspissated material in the small ducts and acini, and no evidence of fibrosis in the early stage of fibrocystic disease. Di Sant' Agnese (1955), on the other hand, examined biopsy material from a patient suffering from fibrocystic disease. This patient still had normal pancreatic enzymes in his duodenal juice. In his case the pancreatic acini and islets were entirely normal, but there was an increase in fibrous tissue and an inflammatory reaction was present in the stroma. It is, of course, impossible to come to any conclusion on such slender evidence, but it may be that an inflammatory 
reaction plays a greater part in the development of the early pancreatic lesion in some patients than has been suspected.

The pancreatic and other lesions in fibrocystic disease have hitherto been regarded as the result of abnormal mucus production, i.e., mucosis. Recently, however, Di Sant' Agnese, Darling, Perera and Shea (1953) have shown that there is an increase in the quantity of electrolytes in the sweat of patients suffering from fibrocystic disease. The sweat glands show no histological change, but are said to be constantly affected in this condition. It would seem, therefore, that exocrine glands other than those producing mucus are affected and the exact pathogenesis of the histological lesions in the pancreas and elsewhere still awaits elucidation.

The liver and gall bladder showed typical changes. The gall bladder was small and fibrosed. It contained only thick mucus. The bile ducts were patent but narrow. No dilated glands were seen. The liver changes were striking. They were characteristically subcapsular in distribution and were very scanty in sections taken from the centre of the liver. They consisted of plugging of small bile ducts in the portal tracts with strongly acidophil secretion. There was proliferation of small bile ducts and a great increase in fibrous tissue in the affected portal tracts. The inspissated secretion had led to dilatation of these small ducts with resulting atrophy to the surrounding liver parenchyma, which had been replaced by fibrous tissue. The appearance was that of biliary cirrhosis which was predominantly focal in distribution. It is possible that this type of lesion plays a part in the development of liver cirrhosis reported by Webster and Williams (1953) in older children with fibrocystic disease. Inflammatory cell infiltration was also a feature of the lesions in the portal tracts and again the polymorphonuclear leucocyte played a prominent part. The density of the cellular infiltration in both the pancreas and the liver was quite exceptional.

The absence of any severe lesion in the intestinal tract was rather surprising. Occasional mucous glands in the stomach, duodenum and ileum were found and these were full of inspissated mucus. Most glands, however, were quite normal apart from rather severe post-mortem change. The colon was heavily loaded with light yellow meconium. This was probably the result of poor biliary secretion and no bile was seen in the gall bladder at necropsy. The consistence of the meconium seemed normal and some had passed through the anal sphincter into the liquor during delivery. This would seem to suggest that the development of meconium ileus is not only dependent on the presence of a severe pancreatic lesion, but also on the disorder of mucus production throughout the alimentary tract. This infant shows that severe pancreatic and liver changes can be present at birth in the absence of comparable lesions in the alimentary tract. Hitherto, cases of fibrocystic disease in the neonatal period have been associated with meconium ileus. In this condition the ileum is grossly distended above and is hypertrophied round inspissated meconium below. The colon is empty and is extremely narrow. By contrast in the present case the ileum was of normal calibre and the colon was full of soft meconium.

The changes in the salivary glands were slight. Unfortunately the sublingual salivary glands were not sectioned. As usual the parotid glands showed no change. The submandibular glands showed some retention of mucus secretion in the acini and the ducts were full of mucus. There was no atrophy of acinar tissue and no fibrosis.

In the respiratory tract the mucous glands of the major bronchi were dilated. The glands were filled with mucus but were not cystic. The changes were minimal and similar to those reported by Nash and Smith (1952). Their significance is doubtful.

There was no doubt that the infant died from the effects of anoxia just before delivery. The foetal heart rate had slowed to $80 / \mathrm{min}$. just after intrapartum haemorrhage occurred. The infant passed meconium into the liquor and there was histological evidence in the lungs of excessive aspiration of liquor amnii. The intrapartum haemorrhage was probably the result of a premature separation of the placenta which was sufficient to interfere with the oxygenation of the infant.

It is interesting to speculate on the course of the disease if the infant had survived the hazards of delivery. The absence of alimentary tract lesions makes it unlikely that intestinal obstruction would have occurred during the neonatal period. The changes in the respiratory tract were very slight and would probably not have been sufficient to encourage the development of infection. The pancreatic lesions were severe and as they are progressive would have led to a very severe nutritional disturbance which would have been soon recognizable. The relationship between focal biliary cirrhosis in the newborn and the development of multilobular cirrhosis in the older child with fibrocystic disease is not yet known. It is possible that the latter condition is not solely the result of malnutrition and protein deficiency.

\section{Summary}

A case of fibrocystic disease of the pancreas in an infant who died during delivery is reported. 
Typical lesions were present in the pancreas and liver. Mild changes were found in the submandibular salivary gland and in the mucous glands of the main bronchi and in some of the mucous glands of the alimentary tract.

There was no evidence of meconium ileus and the meconium was of normal consistence.

An unusual feature of the lesions in the pancreas was the intense inflammatory reaction. The inflammatory cells were mainly polymorphonuclear leucocytes. A brisk inflammatory reaction was also present in the portal tracts in the liver.
I am indebted to Professor J. McLure Browne for access to clinical records and to Mr. E. Clark of this laboratory for the photomicrographs.

\section{REFERENCES}

Andersen, D. H. (1938). Amer. J. Dis. Child., 56, 344.

Bodian, M. (1952). Fibrocystic Disease of the Pancreas. London. Di Sant' Agnese, P. A. (1955). Pediatrics, 15, 683.

- Darling, R. C., Perera, G. A. and Shea, E. (1953). Ibid., 12, 549 .

Farber, S. (1944). Arch, Path. (Chicago), 37, 238.

Fisher, O. D. (1954).Archives of Disease in Childhood, 29, 262.

Levy, E. (1951). Ibid., 26, 335.

Nash, F. W. and Smith, J. F. (1952). Ibid., 27, 73.

Webster, R. and Williams, H. (1953). Ibid., 28, 343 